(c) 2020 Universidad Nacional Autónoma de México, Facultad de Estudios Superiores Zaragoza.

Este es un artículo Open Access bajo la licencia CC BY-NC-ND (http://creativecommons.org/licenses/by-nc-nd/4.0/).

TIP Revista Especializada en Ciencias Químico-Biológicas, 23: 1-13, 2020.

DOI: $10.22201 /$ fesz.23958723e.2020.0.211

\title{
Compósitos en estado hidrogel con aplicación en la adsorción de metales pesados presentes en aguas residuales
}

\author{
Nidia G. Burciaga-Montemayor, Jesús A. Claudio-Rizo, \\ Lucía F. Cano-Salazar, Antonia Martínez-Luévanos y Patricia Vega-Sánchez \\ Depto. de Materiales Avanzados, Facultad de Ciencias Químicas, Universidad Autónoma \\ de Coahuila. Boulevard Venustiano Carranza e Ing. José Cárdenas Valdés s/n, \\ Colonia República, Saltillo 25280, Coahuila, México. E-mail: jclaudio@uadec.edu.mx
}

\begin{abstract}
RESUMEN
La contaminación por metales pesados es un problema, que hoy en día no se ha logrado disminuir. Por esta razón, es necesario innovar constantemente las técnicas tradicionales con el fin de aplicar procesos eficientes que ayuden a remover los contaminantes e incluso recuperarlos para ser reincorporados a procesos productivos. En este contexto, la adsorción es una técnica tan versátil, que es viable su aplicación con materiales de diferentes características. Entre los materiales que han resultado adsorbentes eficientes, se encuentran las partículas inorgánicas y los polímeros/biopolímeros. Estos componentes por si solos presentan capacidades adsorbentes aceptables, pero en los últimos años se ha explorado la generación de matrices poliméricas en estado hidrogel reforzadas con materiales inorgánicos o mezclas de redes poliméricas generando compósitos, para mejorar o incrementar la capacidad de adsorción. Los hidrogeles compósitos conjugan una adsorción eficaz, buena área superficial específica y de fácil aplicabilidad, por lo que representan una gran alternativa para la disminución de los iones de metales pesados presentes en los ecosistemas acuáticos. Por este motivo, es la presente revisión de los materiales con propiedades adsorbentes, las estrategias para generar compósitos en estado hidrogel y sus propiedades adaptadas para la adsorción de iones de metales pesados, así como los retos y las áreas de oportunidad implícitos en esta generación de materiales innovadores.
\end{abstract}

Palabras Clave: metales pesados, adsorción, biopolímeros, hidrogeles compósitos.

Hydrogel composites with application in the remotion of heavy metals present in wastewater

\begin{abstract}
Heavy metal pollution is a problem, that to date has not been mitigated. For this reason, it is necessary to constantly innovate among traditional techniques in order to be able to apply efficient processes in such a way that contaminants can be removed or even recovered to be reincorporated into productive processes. In this context, adsorption is such a versatile technique that its application with materials of different characteristics is viable. Among the materials that have been characterized as efficient adsorbents, are inorganic particles and polymers/biopolymers. These components by themselves have acceptable adsorbent capacities, but in recent years the generation of polymeric matrices in the hydrogel state reinforced with inorganic materials or mixtures of polymeric networks generating composites has been explored to improve or increase the adsorption capacity. Composite hydrogels combine effective adsorption, high specific surface area and easy applicability, so they represent a great alternative for the elimination of heavy metal ions present in aquatic ecosystems. For this reason, the review of the state of the art of materials with adsorbent properties is carried out, as well as the strategies to generate composites in a hydrogel state with properties adapted for the adsorption of heavy metal ions, also contemplating the challenges and areas of opportunity related to generation of this type of innovative materials.
\end{abstract}

Key words: heavy metals, adsorption, biopolymers, hydrogel composites.

Artículo recibido el 11 de noviembre del 2019.

Artículo aceptado el 06 de marzo del 2020. 


\section{INTRODUCCIÓN}

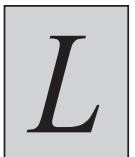

a contaminación del agua es en la actualidad un problema en el que están centradas diversas investigaciones científicas, debido a la vital importancia de este recurso para el desarrollo de la vida en el planeta, por lo que es necesario desarrollar procesos, productos o protocolos para reducir al máximo las implicaciones negativas de las actividades antropogénicas que provocan un daño directo al medio ambiente. Hoy en día los esfuerzos se encuentran enfocados en remediar el deterioro de los ecosistemas $\mathrm{y}$ trabajar para reducir los efectos negativos provocados por los procesos productivos.

En las últimas décadas, los científicos a nivel global han desarrollado diversas técnicas para el tratamiento de efluentes contaminados, sin embargo, aún existen varios problemas que no se han logrado eliminar o reducir. En este contexto, existen algunos contaminantes que, por su naturaleza persistente, son difíciles de remover, estos son los iones de metales pesados, ya que su presencia en el medio ambiente altera de manera instantánea los ecosistemas y por consiguiente a los seres vivos. Muchos de estos contaminantes provienen de forma directa de procesos metalúrgicos o industriales, ejemplos de estos son: plomo, mercurio, cromo, cadmio, arsénico, zinc, entre otros. Al ser México un país rico en yacimientos minerales, no es de extrañar que la minería además de ser uno de los procesos productivos más rentables, también sea uno de los más contaminantes, ya que derivado de la extracción de minerales como el oro y la plata, principalmente, se liberan al medio ambiente diversos residuos que se encuentran asociados a estos metales. En lo que se refiere al plomo puede ser encontrado en su forma mineral (galena, anglesita y curosita) acompañando a otros metales de interés económico, como la plata, y éstos al ser sometidos a procesos de lixiviación generan como subproducto iones de plomo ( $\mathrm{Pb}$ (II) y $\mathrm{Pb}(\mathrm{IV})$ ), que liberados al ambiente terminan por depositarse en el suelo, las plantas y los mantos acuíferos, ya sea superficiales o subterráneos. El arsénico se encuentra en depósitos minerales naturales a profundidades mayores de $800 \mathrm{~m}$, donde debido a la explotación minera, es liberado y entra en contacto con el agua presente en los mantos freáticos. Este metaloide además de ser un bioelemento esencial para muchos organismos es empleado en diversos procesos para generar fertilizantes, pesticidas, pigmentos y pirotecnia, entre otros.

Para poder resolver estas complicaciones, se han desarrollado materiales con diferentes características fisicoquímicas, que pueden retener a los iones metálicos y en algunos casos recuperarlos para que sean reutilizados. Como ejemplo de eficiencia para este propósito son los hidrogeles basados en polímeros con redes entrecruzadas que han mostrado capacidades super-adsorbentes y al entrar en contacto con el contaminante en un medio acuoso son capaces de retenerlo o liberarlo bajo condiciones específicas. Pero además de este tipo de materiales, se ha comenzado a experimentar en la incorporación de otros componentes de carácter inorgánico como las nanopartículas, el carbón activado, el grafeno y las mezclas con diferentes tipos de polímeros naturales $\mathrm{y} / \mathrm{o}$ sintéticos, para desarrollar compósitos en estado hidrogel, con la finalidad de poder potenciar su capacidad de adsorción y controlar sus propiedades.

Los biopolímeros son una opción viable para conformar estos hidrogeles compósitos, dado que se encuentran de forma abundante en la naturaleza, por lo que se pueden obtener y procesar de forma eficiente, mostrando capacidad de biodegradación y crecimiento de microorganismos que puedan contribuir en la transformación de los iones adsorbidos mediante estrategias biotecnológicas. Algunos biopolímeros que se han utilizado con más frecuencia son: el quitosano, la celulosa, el alginato y el colágeno, ya que presentan buenas propiedades fisicoquímicas; como el hinchamiento y la degradación dependientes del $\mathrm{pH}$, permitiendo de esta forma que el contaminante sea retenido, ya sea de forma temporal (fisisorción) o permanente (quimisorción). Investigaciones recientes en este campo han demostrado la eficiencia de conjuntar materiales poliméricos tanto naturales como sintéticos y fases inorgánicas para diseñar hidrogeles compósitos con capacidad de adsorción controlada (Figura 1), por lo que resulta de suma importancia profundizar en las investigaciones documentadas sobre estos procesos y de esta forma, continuar innovando el diseño de este tipo de compósitos en estado hidrogel que por sus características específicas y de selectividad, podrían representar una estrategia, como ya se mencionó, eficiente para el tratamiento de agua contaminada con iones de metales pesados. Por lo tanto, esta revisión tiene como objetivo dar a conocer las propiedades adsorbentes de iones metálicos y su combinación para la generación de hidrogeles compósitos con propiedades adsorbentes mejoradas, los cuales pueden resultar viables para la aplicación en remediación de efluentes contaminados con iones metálicos.

\section{ANTECEDENTES}

La contaminación del agua por metales pesados es un problema en estudio por décadas, debido al aumento indiscriminado en las actividades antropogénicas que ha desequilibrado al medio ambiente afectando la salud humana.

El término metal pesado es un término general que se aplica a un grupo de metales y metaloides con densidad atómica mayor de $4 \mathrm{~g} / \mathrm{cm}^{3}$ (Edelstein \& Ben-Hur, 2018) y su presencia en el medio ambiente como se mencionó anteriormente, corresponde en gran medida a las actividades mineras e industriales, aguas residuales y efluentes domésticos (Waheed, Mansha \& Ullah, 2018).

El proceso de adsorción de los iones de metales pesados está determinado por la afinidad del metal a las biomoléculas, que 


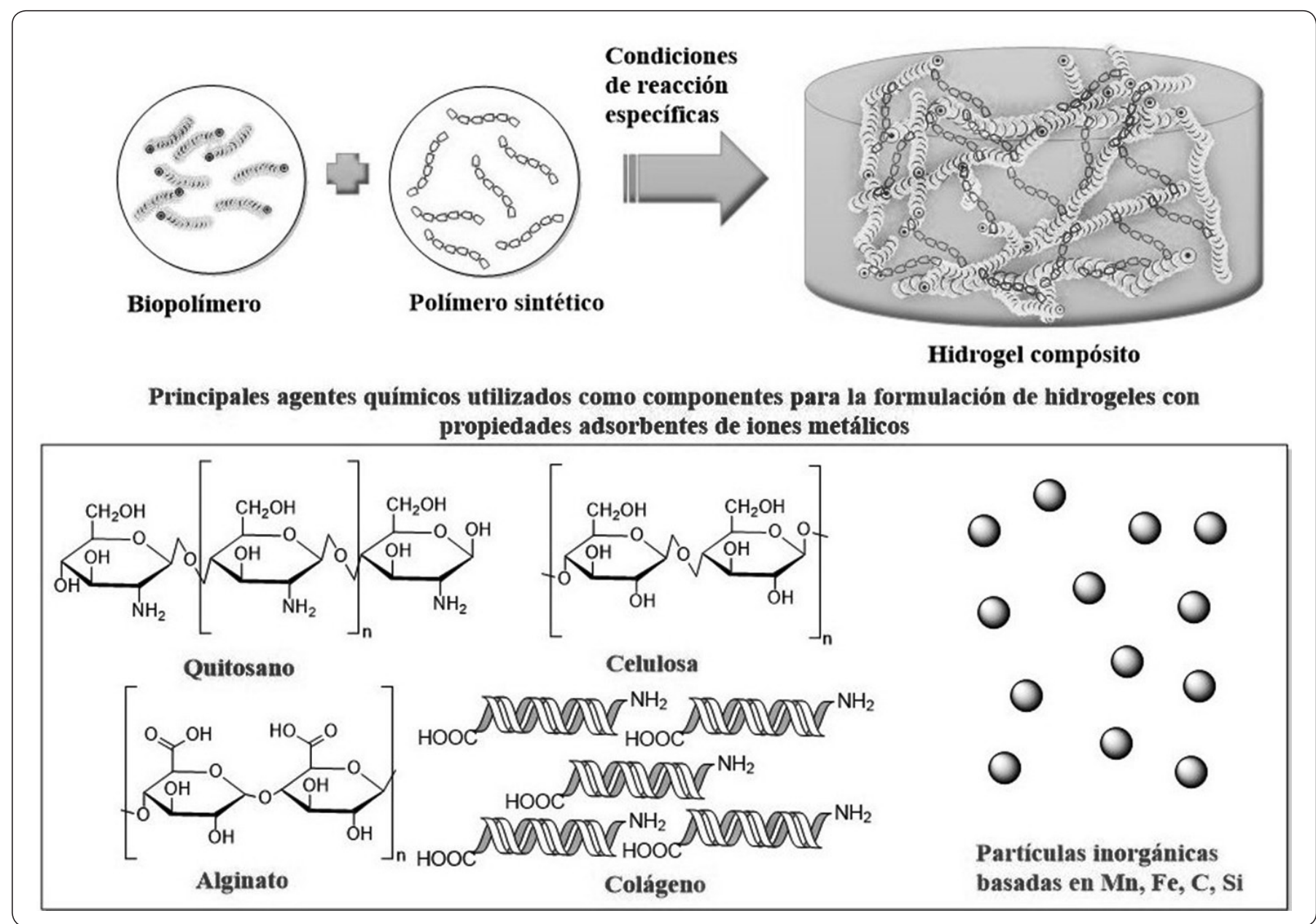

Figura 1. Esquema representativo para la generación de hidrogeles compósitos para la adsorción de iones de metales pesados. Elaboración personal.

contienen grupos funcionales específicos comportándose como ligantes y asociándose a los metales por interacciones físicas o químicas. Nierboer y Richardson clasificaron esta afinidad de los metales en tres grupos: clase A (metales alcalinos, alcalinotérreos, lantánidos, actínidos, Al, Sc eY) con afinidad a ligantes ricos en oxígeno, como los grupos éster, carboxilatos, fosfatos y carbonilos; la clase intermedia o de frontera (metales del primer periodo de transición, metaloides, $\mathrm{Zn}$ y Cd) que presentan afinidad hacia moléculas que contienen grupos con nitrógenos nucleofílicos, como azida, amino, amida y finalmente la clase $\mathrm{B}(\mathrm{Rh}, \mathrm{Pd}, \mathrm{Ag}, \mathrm{Ir}, \mathrm{Pt}, \mathrm{Au}, \mathrm{Hg}, \mathrm{Tl}, \mathrm{Pb}, \mathrm{Bi}$ ), que interactúan con ligantes cianuro, carbaniones, $\mathrm{CO}$ (monóxido de carbono), sulfuro, tioéter y arsinas orgánicas (Nierboer \& Richardson, 1980). Las interacciones pueden ser aprovechadas para el diseño de hidrogeles compósitos y garantizar los procesos de adsorción de los iones metálicos.

La contaminación del agua por iones de metales pesados como: plomo, cadmio, mercurio, cromo y arsénico, son los que representan un mayor peligro para el medio ambiente. Lo anterior debido a la alta toxicidad que presentan, la acumulación de los mismos y el hecho de que no son biodegradables, es la mezcla de características que los hace peligrosos para los organismos vivos (Wen, Fang \& Zeng, 2018). Algunos de los efectos adversos que éstos pueden causar en la salud humana (a muy bajas concentraciones) son enfermedades vasculares y de la piel, daño renal, artritis, desorden cerebral, depresión e incluso cáncer (Burakov et al., 2018). Estos iones metálicos están disueltos en el agua debido a las actividades antropogénicas que emplean elementos como el $\mathrm{Pb}, \mathrm{As}, \mathrm{Cd}, \mathrm{Hg}$, entre otros, generando efluentes que contaminan los mantos acuíferos.

Por lo anterior, la comunidad científica global ha centrado sus esfuerzos en desarrollary/o mejorar técnicas para el tratamiento, remoción o recuperación de estos componentes dañinos para el medio ambiente y la salud. Existen diferentes métodos que están siendo utilizados para la eliminación de metales pesados en el agua, como una gran oportunidad para remediar la huella que se ha generado durante décadas en el medio ambiente. En consideración a la gran cantidad de áreas contaminadas en el 
planeta, es relevante llevar a cabo una revisión de algunas de las técnicas más utilizadas para la remoción/recuperación de los metales pesados, así como las características de algunos materiales que están siendo aplicados en estas técnicas.

Contaminación del AgUa POR METALes PeSAdos Como se ha mencionado, la contaminación del agua debido a la liberación de iones de metales pesados en el medio ambiente es un problema de preocupación mundial; una de las principales fuentes de metales pesados son los desechos de la industria química moderna, como: la fabricación de enchapados y baterías, obtención de fertilizantes, la minería, fabricación de papel, síntesis de pesticidas, utilización de combustibles fósiles y la fabricación de plásticos, entre otros (Carolin, Kumar, Saravanan, Joshiba \& Naushad, 2017). Debido a que estos iones metálicos tienden a acumularse en los organismos vivos, son una gran amenaza para la salud humana. Dentro de los efectos tóxicos de los metales pesados se puede mencionar: el aumento en la presión arterial, problemas del habla, fatiga, insomnio, comportamiento agresivo, reacciones alérgicas, mal funcionamiento de diferentes órganos, pérdida de memoria y desarrollo de cáncer. Los efectos de estos metales en la salud se pueden apreciar a muy bajas concentraciones en el organismo por esto la Agencia de Protección Ambiental de Estados Unidos (EPA, por sus siglas en inglés) y la Organización Mundial de la salud (WHO), han establecido límites permisibles de esos contaminantes en el agua potable (Tabla I).

Tabla I. Límites permisibles de metales pesados en agua potable. Adaptada de (Ihsanullah et al., 2016).

\begin{tabular}{|c|c|c|}
\hline Contaminante & EPA (mg/L) & WHO (mg/L) \\
\hline $\mathrm{Pb}(\mathrm{II})$ & 0,015 & 0,01 \\
\hline $\mathrm{Cr}(\mathrm{III})$ & 0,1 & 0,05 \\
\hline $\mathrm{Cd}(\mathrm{II})$ & 0,005 & 0,003 \\
\hline $\mathrm{As}(\mathrm{IV})$ & 0,010 & 0,01 \\
\hline $\mathrm{Hg}(\mathrm{II})$ & 0,002 & 0,006 \\
\hline $\mathrm{Cu}(\mathrm{II})$ & 1,3 & 2 \\
\hline $\mathrm{Zn}(\mathrm{II})$ & 5 & 3 \\
\hline
\end{tabular}

Como se puede apreciar los límites permisibles del contenido de metales en el organismo es muy baja para los adversos efectos en los seres vivos. En la Tabla II, se muestran las principales fuentes de contaminación por algunos metales pesados y las técnicas que han sido utilizadas para la remediación del problema. Es evidente que la contaminación por metales pesados ha llegado a niveles preocupantes, y la solución está directamente relacionada con la eficiencia, disponibilidad y costo de las técnicas y materiales que se están utilizando para la remediación.

\section{Métodos de Tratamiento}

Varios métodos son utilizados para combatir la presencia de iones de metales pesados en el agua. La selección de alguna técnica en particular dependerá de varios factores entre los que destacan la concentración del contaminante y el costo del método. Las técnicas convencionales para la remoción de metales pesados son: oxidación-reducción, biorremediación, ósmosis inversa, tratamiento electroquímico, coagulación, ablandamiento con cal, precipitación, filtración por membranas, intercambio iónico y adsorción (Tabla II).

Tabla II. Fuentes de contaminación y técnicas de tratamiento de algunos metales pesados (Ihsanullah et al., 2016).

\begin{tabular}{|c|c|c|}
\hline Metal & $\begin{array}{c}\text { Fuentes de } \\
\text { Contaminación }\end{array}$ & $\begin{array}{l}\text { Técnicas de } \\
\text { tratamiento }\end{array}$ \\
\hline Plomo. & $\begin{array}{l}\text { - Pinturas. } \\
\text { - Pesticidas. } \\
\text { - Emisiones de } \\
\text { automóviles. } \\
\text { - Minería. }\end{array}$ & $\begin{array}{l}\text { - Ósmosis inversa. } \\
\text { - Intercambio iónico. } \\
\text { - Cementación. } \\
\text { - Precipitación química. } \\
\text { - Adsorción. }\end{array}$ \\
\hline Arsénico. & $\begin{array}{l}\text { - Pesticidas. } \\
\text { - Fungicidas. } \\
\text { - Agua geotérmica y } \\
\text { desgaste de piedras } \\
\text { volcánicas. }\end{array}$ & $\begin{array}{l}\text { - Coagulación. } \\
\text { - Intercambio iónico. } \\
\text { - Alúmina activada. } \\
\text { - Micro o ultrafiltración. } \\
\text { - Adsorción. }\end{array}$ \\
\hline Mercurio. & $\begin{array}{l}\text { - Depósitos } \\
\text { minerales. } \\
\text { - Pesticidas. } \\
\text { - Baterías. } \\
\text { - Industria del papel. }\end{array}$ & $\begin{array}{l}\text { - Filtración con } \\
\text { membrana. } \\
\text { - Biorremediación. } \\
\text { - Intercambio iónico. } \\
\text { - Precipitación. } \\
\text { - Adsorción. }\end{array}$ \\
\hline
\end{tabular}

De estos métodos, la adsorción es la más versátil y ampliamente usada para la remoción de metales pesados en el agua. Las ventajas que presenta son muchas, como operación simple, bajo costo, buena tolerancia al $\mathrm{pH}$ y capacidad de tratamiento industrial. Adicionalmente, tiene gran eficiencia y selectividad lograda por la funcionalización de los materiales. Estas características son deseadas en el tratamiento de aguas a nivel industrial (Xue, Cheng, Shi, Ma \& Zheng, 2017).

\section{Adsorción}

La adsorción se define como un proceso que ocurre cuando un gas o líquido se acumulan en la superficie de un sólido o líquido (adsorbente), formando una película molecular o atómica (adsorbato). El actor principal de esta técnica es el adsorbente, el cual se selecciona dependiendo del metal presente, la concentración de éste, la eficiencia y la capacidad de adsorción selectiva para el contaminante que se desee retener. Adicionalmente, el adsorbente no debe de ser tóxico, debe tener buena relación costo/beneficio, ser de fácil obtención y que pueda regenerarse o reutilizarse (Singh, Nagpal, Agrawal \& Rachna, 2018). Es por esta razón que, en años recientes una gran cantidad de investigaciones giran alrededor de la ciencia e ingeniería de materiales adsorbentes que cumplan con los requisitos 
para ser utilizados en la técnica de adsorción. La adsorción es más prometedora y atractiva, y ha sido ampliamente utilizada debido a sus características de rentabilidad, operación simple, menos contaminación secundaria y alta eficiencia. Durante los últimos años, se han llevado a cabo diversas investigaciones sobre el uso de diferentes adsorbentes para la purificación de aguas residuales (Singh et al., 2018).

\section{MATERIALES ADSORBENTES}

Las características fisicoquímicas que definen a un material adsorbente son su área superficial (relacionada con el área total que ocupa la superficie del material) y su capacidad adsorbente (relacionada con los sitios activos que pueden ser utilizados para retener especies químicas mediante interacciones fisicoquímicas). En los últimos años, se han desarrollado múltiples alternativas para reducir el impacto de la contaminación por iones de metales pesados en el agua; materiales inorgánicos como los óxidos metálicos de dimensiones micrométricas y nanométricas, incluidos óxidos férricos, de manganeso, aluminio, titanio, magnesio y cerio, proporcionan una gran área superficial y una afinidad específica por adsorción de metales pesados de sistemas acuosos; donde estos materiales en escala nanométrica muestran propiedades redox y fotocatalíticas mejoradas, representando estrategias eficientes para el tratamiento de los iones adsorbidos.

Otra metodología que ha sido constantemente estudiada con la finalidad de mejorar su desempeño en la adsorción, es el uso de carbón activado modificado. Esta estrategia está en función de varios factores, incluyendo el $\mathrm{pH}$, la temperatura y la concentración de contaminantes, donde las propiedades del adsorbente determinan el mecanismo de eliminación. En cuanto a la adsorción de metales pesados son efectivos los carbones activados modificados con grupos ácidos o básicos (por ejemplo, incluyendo grupos fenólicos, carboxilo, lactona e hidroxilo en su superficie), sin embargo, la eficiencia de esta técnica se limita a soluciones monocomponentes (soluciones modelo) y representa altos costos de operación para la recuperación de las especies adsorbidas (Burakov et al., 2018; Shams et al., 2016). Las nanopartículas magnéticas como las nanopartículas de óxido de hierro, también han mostrado capacidad óptima para la eliminación de: $\mathrm{Pb}$ (II), $\mathrm{Hg}$ (II), $\mathrm{Cu}$ (II) y Co (II) bajo la acción de campos magnéticos (Wanna et al., 2016). En la Tabla III se resumen los materiales en fase inorgánica que han sido empleados como adsorbentes tradicionales de metales pesados, indicando los principales procesos de síntesis de esos materiales, los iones metálicos adsorbidos, su capacidad de adsorción y las ventajas que sus sistemas presentan. Aunque los materiales en fase inorgánica tienen buena capacidad adsorbente de iones metálicos, la principal limitante en su uso efectivo es la generación de lodos de desecho, ya que requieren procesos de separación y purificación que involucran altos costos temporales y económicos.
Apesar de que los adsorbentes tradicionales siguen presentando buenas características físicas y químicas, se busca el desarrollo de nuevos materiales que complementen el funcionamiento adsorbente de las fases inorgánicas comúnmente usadas, evitando la generación de lodos y el uso de técnicas de microfiltración y centrifugación para la recuperación del material adsorbente. En la búsqueda de la optimización de las capacidades de adsorción se ha optado por investigar las propiedades de diferentes materiales, algunos que provienen de residuos tan comunes como la piel de frutos y vegetales, incluso desechos provenientes del procesamiento de alimentos de origen animal (Colina et al., 2014). Estos estudios han demostrado que los biopolímeros constituyentes de estos residuos, poseen grandes capacidades adsorbentes por si solos (mayores a $80 \mathrm{mg} / \mathrm{g}$ ), pero con algunas deficiencias mecánicas y de estabilidad térmica, limitando su aplicación en estrategias de periodos largos de uso y medios hidrolíticos severos (pH ácidos y/o alcalinos), lo que conlleva a la utilización de agentes estabilizadores que alteran sus capacidades de adsorción (Balleño, Ríos, Aranda, Morales \& Katime, 2016). Los biopolímeros como la celulosa, el almidón, el colágeno, el quitosano, la gelatina y el alginato han mostrado altas capacidades adsorbentes a diversos iones metálicos, sin embargo, su pobre estabilidad mecánica y rápida velocidad de degradación limitan su aplicación como materiales adsorbentes (Noor, Othman, Mubarak \& Abdullah, 2017). Estrategias que contemplan la mejora de estas propiedades de los biopolímeros involucran el uso de agentes de entrecruzamiento químico, donde los enlaces de reticulación formados controlan su desempeño mecánico y su velocidad de degradación, sin embargo, también alteran su morfología típica y capacidad adsorbente. La formación de sistemas de doble red polimérica usando mezclas de polímeros representa una manera efectiva de controlar la mecánica, degradación y capacidad adsorbente de los biopolímeros. El uso de los biopolímeros como materiales adsorbentes de iones de metales pesados representa una estrategia importante para la eliminación y/o tratamiento de las especies adsorbidas. En este sentido, los biopolímeros pueden ser degradados por enzimas específicas para la liberación de los iones adsorbidos. Estrategias biotecnológicas involucran el uso de microorganismos con capacidad metabólica para asimilar los iones metálicos adsorbidos, representando estrategias innovadoras de biorremediación de cuerpos acuosos contaminados. El uso de polímeros sintéticos como el poliacrilato, la poliacrilamida, el poliestireno y el polipropileno, entre otros, se ve limitado debido a que los materiales con capacidad adsorbente basados en ellos muestran una degradación pobre, limitando la recuperación rápida de las sustancias adsorbidas y representando un tema de controversia por la generación de residuos con carácter tóxico. Sin embargo, algunas estrategias involucran el uso de estos componentes poliméricos en proporciones pequeñas, cuando se mezclan con polímeros naturales a fin de regular la mecánica y degradación de estos últimos. 
Tabla III. Materiales inorgánicos adsorbentes de iones de metales pesados.

\begin{tabular}{|c|c|c|c|c|}
\hline $\begin{array}{c}\text { Material } \\
\text { Inorgánico }\end{array}$ & Síntesis & \begin{tabular}{|c|}
$\begin{array}{c}\text { Metal adsorbido } \\
\text { y eficiencia }\end{array}$ \\
\end{tabular} & Ventajas & Referencia \\
\hline $\begin{array}{l}\text { Carbón } \\
\text { Activado. }\end{array}$ & $\begin{array}{l}\text { - Activación térmica } \\
\text { simple en atmósfera } \\
\text { de nitrógeno. } \\
\text { - Radiación de } \\
\text { microondas. } \\
\text { - Pirólisis. } \\
\text { - Activación química. } \\
\text { - Activación Física. }\end{array}$ & 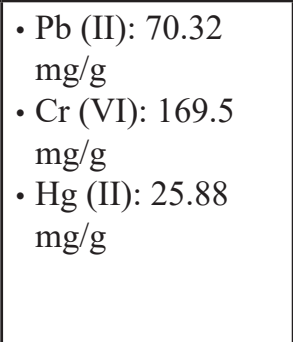 & $\begin{array}{l}\text { - Buena capacidad adsorbente. } \\
\text { - Amplia disponibilidad en la } \\
\text { naturaleza de los precursores. } \\
\text { - Diversas rutas de síntesis. } \\
\text { - Aplicabilidad simple y } \\
\text { variada. }\end{array}$ & (González-García, 2018). \\
\hline Zeolita. & $\begin{array}{l}\text { - Síntesis hidrotermal. } \\
\text { - Método de } \\
\text { microemulsión } \\
\text { inversa. }\end{array}$ & $\begin{array}{l}\cdot \mathrm{Pb}(\mathrm{II}): 510 \mathrm{mg} / \mathrm{g} \\
\cdot \mathrm{Cu}(\mathrm{II}): 170 \\
\mathrm{mg} / \mathrm{g} \\
\cdot \begin{array}{l}\mathrm{Hg}(\mathrm{II}): 25.88 \\
\mathrm{mg} / \mathrm{g}\end{array}\end{array}$ & $\begin{array}{l}\text { - Aplicaciones diversas. } \\
\text { - Buen rendimiento. } \\
\text { - Capacidad de encapsular } \\
\text { grupos activos. } \\
\text { - Propiedades antimicrobianas. } \\
\text { - Económicamente viable. }\end{array}$ & $\begin{array}{l}\text { (Collins, Rozhkovskaya, } \\
\text { Outram \& Millar, 2020; Hong, } \\
\text { 2019). }\end{array}$ \\
\hline Grafeno. & $\begin{array}{l}\text { - Exfoliación en fase } \\
\text { líquida. } \\
\text { - Deposición química } \\
\text { de vapor. } \\
\text { - Exfoliación mecánica. } \\
\text { - Descarga de Arco. } \\
\text { - Exfoliación redox. } \\
\text { - Síntesis orgánica total. }\end{array}$ & 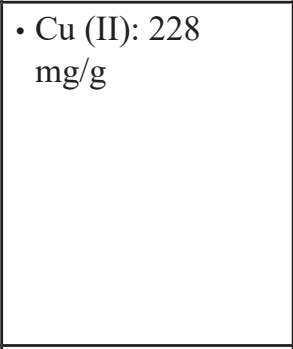 & $\begin{array}{l}\text { - Fácil funcionalización con } \\
\text { otros materiales. } \\
\text { - Diversas rutas de síntesis. } \\
\text { - Capacidad regenerativa. }\end{array}$ & $\begin{array}{l}\text { (Baig, Ihsanullah, Sajid \& } \\
\text { Saleh, 2019; Lee, 2019). }\end{array}$ \\
\hline Manganita. & $\begin{array}{l}\text { - Sol-gel. } \\
\text { - Sinterización por } \\
\text { plasma por chispa. }\end{array}$ & \begin{tabular}{|l|} 
- $\mathrm{Pb}$ (II): $98 \mathrm{mg} / \mathrm{g}$ \\
- $\mathrm{Cd}$ (II): $6.8 \mathrm{mg} / \mathrm{g}$ \\
- As (IV): $60 \mathrm{mg} / \mathrm{g}$
\end{tabular} & $\begin{array}{l}\text { - Mineral natural de bajo costo. } \\
\text { - Disponibilidad natural. } \\
\text { - Variabilidad de rutas de } \\
\text { síntesis. } \\
\text { - Propiedades adsorbentes para } \\
\text { diversos iones metálicos. }\end{array}$ & $\begin{array}{l}\text { (Ayadi et al., 2018; Crisóstomo } \\
\text { et al., 2007; Siddiqui, Naushad } \\
\text { \& Chaudhry, 2019; Sönmezay, } \\
\text { Öncel \& Bektaş, 2012; } \\
\text { Crisóstomo et al., 2007; Ayadi, } \\
\text { 2018; Sönmezay et al., 2012; } \\
\text { Siddiqui et al., 2019). }\end{array}$ \\
\hline
\end{tabular}

La combinación de fases inorgánicas en matrices biopoliméricas es una alternativa innovadora para potencializar la capacidad adsorbente de los componentes y mejorar sus propiedades mecánicas y la velocidad de degradación, sin embargo, el reto es generar materiales que tengan una estructura estable para que puedan tener un proceso de adsorción controlado. Con esto en mente, la generación de hidrogeles basados en redes poliméricas reticuladas constituidas por biopolímeros representa una alternativa potencial para este propósito. Un hidrogel es una red polimérica altamente entrecruzada que tiene la capacidad de adsorber grandes masas de agua, generando sistemas $3 \mathrm{D}$ estables que pueden ser empleados para la difusión de especies químicas hacia dentro o fuera de esta matriz. Este tipo de matrices en estado hidrogel podrían ser acopladas con fases inorgánicas y/o poliméricas para generar nuevos materiales compósitos con capacidad de adsorción mejorada, eliminando la generación de lodos y que el material sea fácilmente removido.

\section{HidRogeles COMPÓSITOS PARA LA ADSORCIÓN DE IONES DE METALES PESADOS}

El hidrogel es una red tridimensional de polímeros reticulados, una de sus características es la alta dispersión en el agua, proporcionando así la entrada de diversos materiales a su interior. Sin embargo, la baja resistencia mecánica, la rápida degradación hidrolítica y la escasa capacidad de recuperación o desahogo del hidrogel limitan su extensa aplicación (Yu et al., 2016). Algunos nuevos hidrogeles con resistencia mecánica mejorada han sido desarrollados, por ejemplo, los hidrogeles polianfolíticos, hidrogeles nanocompósitos ehidrogeles de doble red. Estos últimos comprenden dos polímeros interpenetrantes y redes reticuladas, constituyendo la estrategia más efectiva para mejorar el módulo de almacenamiento de los hidrogeles. Además, en los hidrogeles de doble red no existen enlaces covalentes entre las cadenas poliméricas, sino más bien las cadenas se mantienen unidas mediante la generación de interacciones dinámicas que incluyen interacción electrostática, 
reticulación iónica y/o enlaces de puente de hidrógeno. Por lo tanto, esta singularidad de los hidrogeles de doble red podría abrir nuevas oportunidades para explorar la posible aplicación de estos materiales como adsorbentes de iones metálicos, ya que al no existir enlaces de reticulación covalentes la degradación del hidrogel es controlada beneficiando la recuperación y/o liberación de las especies adsorbidas (Figura 2).

Dentro de los hidrogeles desarrollados a partir de biopolímeros es importante destacar el trabajo realizado al sintetizar un hidrogel de doble red a base de almidón y poliacrilato, para la eliminación de metales pesados en medios acuosos, la técnica resultó ser rápida y eficiente. Esta técnica fue probada en aguas modelo con alto contenido de Cd (II) (aproximadamente 180 $\mathrm{mg} / \mathrm{L}$ ), tal fue la eficiencia del proceso, que sólo con $1 \mathrm{~g} / \mathrm{L}$ del adsorbente se llegó rápidamente al equilibrio, logrando la eliminación completa del contaminante. Otro de los beneficios de esta técnica radica en la posterior desorción del contaminante y su recuperación para la reintegración a procesos industriales (Xu et al., 2015), ya que los hidrogeles pueden ser degradados rápidamente para la eliminación de las especies adsorbidas modificando el $\mathrm{pH}$ del medio.

Los polímeros insolubles formadores de hidrogeles y partículas inorgánicas se pueden ensamblar para formar hidrogeles compósitos, que han atraído una atención especial durante la última década. Algunos minerales de arcilla como esmectita, montmorillonita natural y sintética, así como hectorita, son utilizados con frecuencia debido a su estructura en capas y propiedades únicas. La estructura dota a estos minerales arcillosos con sobresaliente intercambiabilidad iónica, adsorción e hinchamiento. Una estrategia para la formación de un innovador hidrogel compósito se basó en la generación de hidrogeles de quitosano acoplados con estos minerales. Este tipo de hidrogel compósito exhibió propiedades mecánicas, ópticas y de hinchamiento extraordinarias (Vieira, Vilela, Becegato \& Paulino, 2018), indicando una capacidad de absorción del $\mathrm{Cr}$ (VI) de $96 \mathrm{mg} / \mathrm{g}$.

Entre los compósitos en estado hidrogel reforzados con nanocompósitos, se puede destacar el ensamble de atapulgita sobre una matriz de poliacrilamida, esta técnica resultó ser selectiva para la recuperación de $\mathrm{Pb}$ (II) y Cu (II) en soluciones acuosas, además uno de los grandes beneficios de este hidrogel radica en la posibilidad de que el material saturado de iones metálicos puede someterse a un proceso de desorción y los iones reintegrados a procesos industriales, lo que hace de este material, un compósito eficiente y amigable con el medio ambiente (Liu, Jiang, Zhu, Guo \& Wang, 2015).

Para el diseño de los hidrogeles compósitos existen varios polímeros que se vuelven una constante en diferentes investigaciones, ya que por sus características, eficiencia o biodisponibilidad, han resultado ser elementos funcionales al momento de sintetizar hidrogeles. El colágeno, el almidón, el alginato, la celulosa y el quitosano, son algunos de los

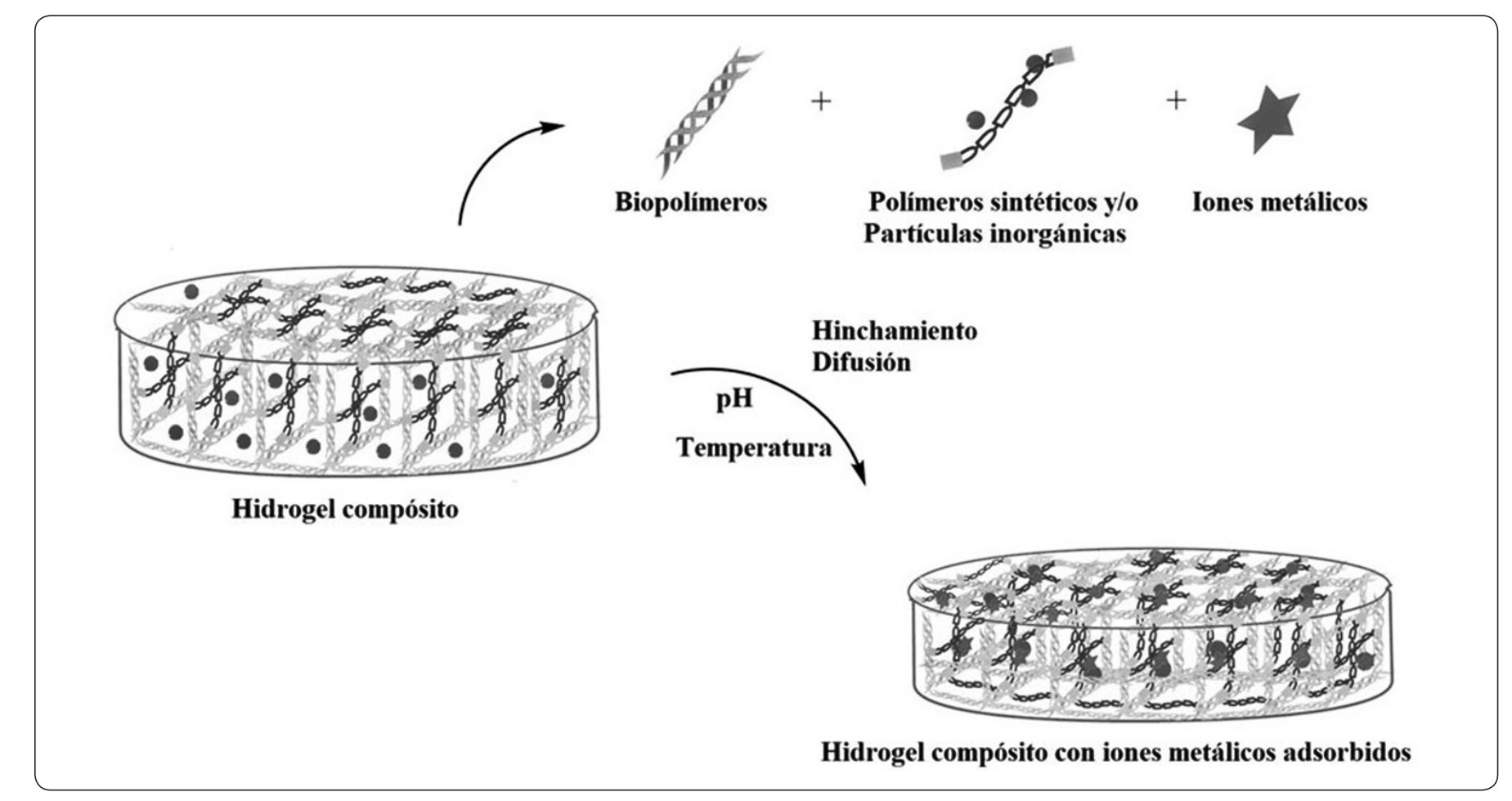

Figura 2. Esquema representativo para la difusión y adsorción de iones metálicos en hidrogeles compósitos. Elaboración personal. 
biopolímeros más utilizados en la síntesis de hidrogeles biodegradables gracias a sus bajos costos, abundancia en la naturaleza y propiedades de adsorción. Dentro de éstos, la celulosa y el quitosano tienen alta disponibilidad natural aunado a los abundantes grupos funcionales hidrofílicos (hidroxilo, carboxilo y/o amino) en su estructura, se han convertido en los materiales por excelencia cuando se trata de hidrogeles adsorbentes (Hong, Okabe, Hidaka \& Hara, 2018b).

El uso de estos biopolímeros en su mayoría va enfocado a lograr fases interpenetradas para la generación de hidrogeles, ya que las propiedades y eficiencia de los materiales poliméricos se ven mejoradas, de esta forma se puede establecer un grado eficiente de entrecruzamiento físico, lo que garantiza un control en la capacidad de hinchamiento y estabilidad del hidrogel (Vera, Pérez \& Sabino, 2016). Algunos biopolímeros tienen cierta afinidad a los iones metálicos, como es el caso de la carboximetil celulosa, la cual presenta gran eficiencia para la adsorción de Cr (VI) y Cd (II) (Corvo, López, León \& Corrales, 2014). La celulosa es un polímero orgánico natural que se encuentra en la mayoría de las plantas vasculares terrestres y ha demostrado su gran capacidad para la eliminación de iones metálicos debido principalmente a la coordinación, el intercambio iónico y la asociación electrostática entre el adsorbente y el adsorbato (Wang et al., 2013a); por lo que resulta ser un material interesante por presentar una cantidad de grupos funcionales en su estructura con capacidad adsorbente.

Otro biopolímero que sigue siendo sumamente utilizado es el quitosano, como segundo amino-polisacárido natural más abundante proveniente de artrópodos y conchas de crustáceos por mencionar: langostas, cangrejos, camarones y sepias, representando un residuo de la industria alimentaria, lo que aporta un carácter sustentable para la generación de hidrogeles adsorbentes basados en este biopolímero. El quitosano, como producto de la desacetilación de la quitina, genera una red polimérica con capacidad de formar hidrogeles interpenetrados con diferentes agentes químicos, generando redes resistentes y con la suficiente área superficial, como para albergar otros componentes, controlando su hinchamiento y degradación; además la presencia de grupos amino e hidroxilo en su estructura le aportan a este biopolímero la capacidad adsorbente de iones metálicos. Ha sido reportada la generación de un hidrogel compósito a base de quitosano acoplado con partículas de sílice, indicando retención eficiente de $\mathrm{Cu}$ (II), transformándose en un material con características óptimas para el tratamiento de efluentes contaminados con metales pesados (Escoda et al., 2013).

El alginato de sodio es otro material que recientemente ha sido funcionalizado con polímeros sintéticos a fin de lograr adsorber contaminantes como iones de metales pesados. El alginato de sodio es un biopolímero rentable y no tóxico, además cuenta con una eficaz capacidad de adsorción gracias a los grupos hidroxilo y carboxilato que se encuentran en su estructura. Wang y colaboradores identificaron en el polímero natural alginato de sodio la viabilidad para ser funcionalizado con vinilo por polimerización por radicales libres produciendo un compósito hidrogel nanoestructurado con gran eficiencia para adsorber metales, en específico Pb (II) (Wang, Zong \& Wang, 2013b).

El uso de residuos basados en colágeno representa otra alternativa para generar hidrogeles compósitos con propiedades adsorbentes mejoradas. El colágeno es la principal proteína constituyente de los tejidos dérmicos, epiteliales y conectivos de los mamíferos. La estructura primaria del colágeno está establecida por la presencia de los aminoácidos prolina e hidroxiprolina y su alto contenido en grupos amino primario, podrían ser sitios para interacciones de quimisorción con iones metálicos. En este sentido, Wang y colaboradores desarrollaron perlas de hidrogel compuestas por colágeno/quitosano/celulosa con la finalidad de adsorber $\mathrm{Cu}$ (II), mediante la unión de los sitios activos de los grupos amino con el $\mathrm{Cu}$ (II). Las redes poliméricas de este sistema de hidrogel se encuentran unidas por enlaces de hidrógeno, lo que permite que la conformación de estos biopolímeros dé como resultado hidrogeles compósitos con amplia compatibilidad entre sus precursores. Estos materiales presentaron un porcentaje de adsorción máximo de $0.47 \mathrm{mmol} / \mathrm{g}$, sin embargo, derivado de este estudio se identificó que al aumentar la proporción de colágeno de 1/1/1 a 2/1/1 se mejoran las propiedades de adsorción del ion $\mathrm{Cu}$ (II) significativamente, por lo que se reafirma la gran capacidad adsorbente de los iones metálicos que presenta el colágeno, abriendo las posibilidades de aplicar materiales con base en este biopolímero para usos industriales ( Wang et al., 2013a). Otro de los trabajos relacionados con lo antes expuesto y que ha sido publicado recientemente, es el presentado por Singha y colaboradores, estos investigadores desarrollaron un proceso de adsorción eficiente en un hidrogel compósito a base de colágeno residual proveniente de las curtidurías, este biopolímero es entrecruzado con caucho natural por acción de reacciones de condensación usando fenol/formaldehído/bisfenol. Este método resultó eficiente y una opción viable para la adsorción de Hg(II) (Roy et al., 2019). En la Tabla IV se muestran los principales sistemas de hidrogeles compósitos diseñados para la adsorción de iones metálicos de transición, indicando la ruta de síntesis de los novedosos materiales, su capacidad adsorbente y las ventajas mostradas para cada sistema.

Los procesos convencionales para la remoción de metales pesados en agua involucran diferentes etapas que representan inversión de tiempo y costo; por un lado el tiempo necesario en técnicas como ósmosis inversa, coprecipitación y sedimentación son parámetros a considerar cuando se emplean; por otra parte la infraestructura requerida para las técnicas de alta eficiencia de remoción como biorremediación, ósmosis inversa, tratamiento electroquímico, filtración por membranas e intercambio iónico son una limitante para que sean aplicadas convencionalmente. En 
Tabla IV. Hidrogeles compósitos para la eliminación de metales pesados en agua.

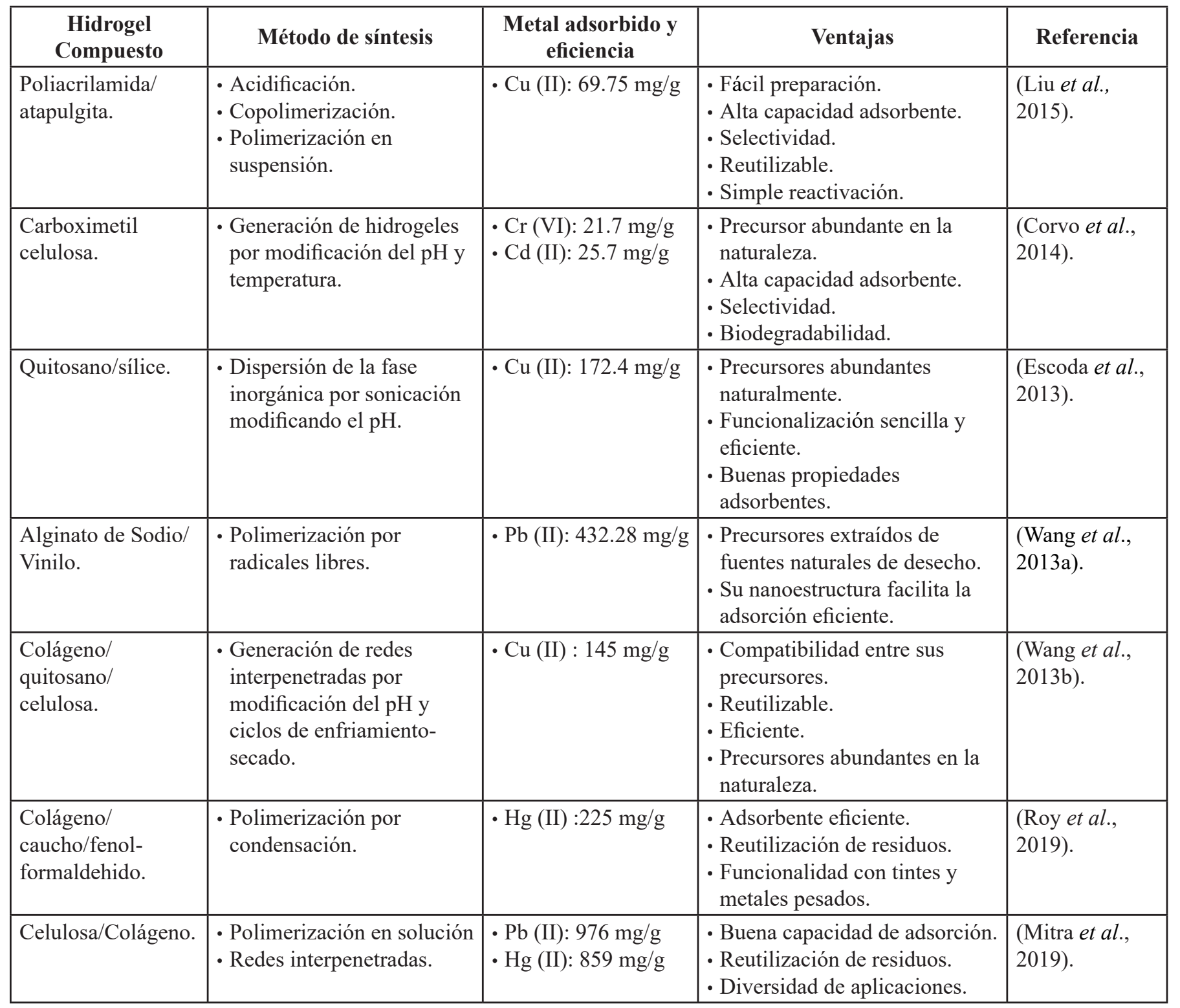

este sentido, los hidrogeles compósitos representan un sistema con facilidad de uso y eficiencia de remoción en tiempos cortos (equilibrios de adsorción son alcanzados en tiempos cortos) y se puede aprovechar la abundancia natural de los biopolímeros para la generación de sistemas de fácil accesibilidad económica. En la Tabla V se resumen las ventajas y desventajas de algunos procesos para remover metales pesados en aguas residuales.

Al analizar la información disponible sobre los adsorbentes que se aplican y se han aplicado para la remediación de aguas contaminadas se tiene una perspectiva real y fundamentada de las causales que aportan un gran valor a los procesos de adsorción por lo cual pueden ser de dos tipos: fisisorción y quimisorción. En la fisisorción interacciones electrostáticas y/o fuerzas de menor grado como puentes de hidrógeno y/o interacciones dipolares son responsables de la adsorción del ion metálico en la superficie del material; en la quimisorción existe la generación de enlaces de coordinación entre los componentes de los adsorbentes y el adsorbato; ambos procesos pueden ser llevados mediante el uso de materiales a base de polímeros/ biopolímeros y compuestos inorgánicos. Estos materiales por sí solos son funcionales, selectivos y con una eficiencia aceptable. Sin embargo, las investigaciones actuales están apostando a la conjunción de diferentes componentes, lo que deriva en la obtención de nuevos compósitos en estado hidrogel con propiedades y eficiencias mejoradas, que abren un panorama alentador para el tratamiento de efluentes con contenidos de iones metálicos, además de que en algunos casos existe la 
Tabla V. Comparación de las técnicas de remoción de iones de metales pesados.

\begin{tabular}{|c|c|c|c|}
\hline $\begin{array}{l}\text { Método de } \\
\text { remoción }\end{array}$ & Ventajas & Desventajas & Referencia \\
\hline Ósmosis inversa. & $\begin{array}{l}\text { - Alta eficiencia de } \\
\text { remoción. }\end{array}$ & $\begin{array}{l}\text { - Infraestructura costosa. } \\
\text { - Selectividad dependiente del potencial eléctrico. } \\
\text { - Sensible a interferencias. }\end{array}$ & (Dialynas, 2008). \\
\hline $\begin{array}{l}\text { Filtración por } \\
\text { membranas. }\end{array}$ & $\begin{array}{l}\text { - Alta eficiencia de } \\
\text { remoción. }\end{array}$ & $\begin{array}{l}\text { - El tiempo de remoción depende de la selectividad } \\
\text { de la membrana al ion metálico. } \\
\text { - Capacidad de uso limitada. } \\
\text { - Selectividad de remoción dependiente de la } \\
\text { naturaleza de la membrana. } \\
\text { - Bajo desempeño a pH ácidos o alcalinos. }\end{array}$ & (Buonomenna, 2013). \\
\hline $\begin{array}{l}\text { Tratamiento } \\
\text { electroquímico. }\end{array}$ & $\begin{array}{l}\text { - Alta eficiencia de } \\
\text { remoción de iones de } \\
\text { metales. }\end{array}$ & $\begin{array}{l}\text { - Infraestructura costosa. } \\
\text { - La naturaleza de los electrodos determinan la } \\
\text { rapidez y eficiencia del proceso. }\end{array}$ & (Wang et al., 2020). \\
\hline $\begin{array}{l}\text { Hidrogeles } \\
\text { compósitos. }\end{array}$ & $\begin{array}{l}\text { - Alta eficiencia de } \\
\text { remoción. } \\
\text { - No requieren } \\
\text { infraestructura costosa. } \\
\text { - Fácil aplicabilidad. }\end{array}$ & $\begin{array}{l}\text { - Generación de subproductos de degradación } \\
\text { tóxicos dependiente de la naturaleza química del } \\
\text { hidrogel. } \\
\text { - Diseño de dispositivos para aplicación en la } \\
\text { remoción a gran escala. }\end{array}$ & (Wang et al., 2013b). \\
\hline
\end{tabular}

posibilidad de recuperación y reincorporación de los iones a procesos productivos (Roy et al, 2019).

Es necesario seguir investigando en el diseño y desarrollo de nuevos sistemas de hidrogeles compósitos que permitan controlar los procesos de adsorción de iones metálicos de una manera rápida y sustentable. El estudio de los procesos de polimerización para generar estas innovadoras matrices en estado hidrogel, así como también la modificación involucrada en esos procesos por el acoplamiento de fases inorgánicas y/o poliméricas, representa un área de oportunidad en la ciencia y la tecnología de los nuevos materiales. Los hidrogeles basados en biopolímeros deben tener una velocidad controlada de degradación y un hinchamiento regulado que no involucre la falla mecánica de la red 3D para promover un proceso de adsorción controlado (Mitra et al. 2019).

Las estrategias enfocadas en controlar estas propiedades deben considerar no alterar la biodegradación natural de los biopolímeros, ya que ésta es una ventaja que puede ser aprovechada para la generación de materiales adsorbentes que puedan ser degradados por estrategias de la biorremediación. En este contexto, los hidrogeles compósitos representan sistemas $3 \mathrm{D}$ donde pueden ser encapsulados microorganismos que en contacto con el ion metálico lo puedan metabolizar para que sea menos tóxico a la salud. De esta manera es de vital importancia mantener la estructura química de los biopolímeros para permitir el desarrollo de estrategias biotecnológicas. El uso de polímeros sintéticos para la generación de hidrogeles compósitos también debe ser balanceado con la finalidad de tener materiales que muestren control en sus propiedades mediante la alteración de factores como la temperatura, el $\mathrm{pH}$ y la concentración de los iones metálicos de interés. Específicamente en México, las industrias petroquímicas, metalúrgicas, asociadas con la formulación de abonos y fertilizantes, así como la elaboración de cuero están asociadas con la contaminación de los mantos acuíferos con iones de metales pesados (Fernández-Constantino et al., 1998). A la fecha se sigue estudiando exhaustivamente para la generación de compósitos en estado hidrogel que presenten un balance entre sus propiedades fisicoquímicas, de degradación y capacidad adsorbente; como un área de investigación prometedora para su aplicación directa en los efluentes industriales o mineros de México, de esta forma se podrá comenzar a reducir el impacto negativo que estas industrias generan día con día en el entorno.

\section{Conclusiones}

La adsorción, como proceso de tratamiento de efluentes contaminados por iones de metales pesados, es sin duda una de las mejores técnicas por sus beneficios, eficiencia y el bajo costo de aplicación. Sin embargo, es necesario desarrollar compósitos en estado hidrogel basados en polímeros/biopolímeros y/o fases 
inorgánicas que permitan incrementar la capacidad de adsorción y sean una estrategia innovadora para la eliminación de iones de metales pesados presentes en cuerpos acuosos. El uso de este tipo de materiales evita la generación de residuos que tengan un potencial carácter tóxico para el ambiente, con una estrategia rápida y eficiente para combatir un problema de carácter nacional y mundial; para ello es necesario seguir investigando el desarrollo de nuevas matrices compuestas en estado hidrogel que presenten modulación en su relación estructura/propiedades para su aplicación potencial como materiales adsorbentes de iones de metales pesados.

\section{Agradecimientos}

La presente investigación se realizó con el apoyo del Cuerpo Académico de Materiales Avanzados adscrito a la Facultad de Ciencias Químicas de la UniversidadAutónoma de Coahuila, por lo que se agradece a esta institución el acceso a la información, la preparación y disponibilidad de los docentes pertenecientes a esta Facultad. Se agradece al Consejo Nacional de Ciencia y Tecnología (CONACYT) por la beca No. 700932.

\section{REFERENCIAS}

Ayadi, F., Ammar, S., Nowak, S., Cheikhrouhou-Koubaa, W., Regaieg, Y., Koubaa, M., Monier, J. \& Sicard, L. (2018). Importance of the synthesis and sintering methods on the properties of manganite ceramics: The example of La 0.7 $\mathrm{Ca} 0.3 \mathrm{Mn}$ 03. Journal of Alloys and Compounds, 759, $52-$ 59. https://doi.org/10.1016/j.jallcom.2018.05.113.

Baig, N., Ihsanullah, Sajid, M. \& Saleh, T.A. (2019). Graphenebased adsorbents for the removal of toxic organic pollutants: A review. Journal of Environmental Management, 244, 370-382. https://doi.org/10.1016/j.jenvman.2019.05.047.

Balleño, A., Ríos, N., Aranda, F. J., Morales, J. A. \& Katime, I. (2016). Hidrogeles de alginato-quitosano y alginato-sulfato de quitosano para la remoción de iones cobre. Revista Iberoamericana de Polimeros, 17(5), 255-265. http://www. ehu.eus/reviberpol/pdf/SEPT16/Balleno.pdf.

Buonomenna, M. G. (2013). Membrane processes for a sustainable industrial growth. RSCAdvances, 3, 5694-5740. https://doi.org/10.1039/C2RA22580H 2.

Burakov, A. E., Galunin, E. V., Burakova, I. V., Kucherova, A. E., Agarwal, S., Tkachev, A. G. \& Gupta, V. K. (2018). Adsorption of heavy metals on conventional and nanostructured materials for wastewater treatment purposes: A review. Ecotoxicology and Environmental Safety, 148, 702-712. https://doi.org/10.1016/j.ecoenv.2017.11.034.

Carolin, C. F., Kumar, P. S., Saravanan, A., Joshiba, G. J. \& Naushad, M. (2017). Efficient techniques for the removal of toxic heavy metals from aquatic environment: A review. Journal of Environmental Chemical Engineering, 5(3), 2782-2799. https://doi.org/10.1016/j.jece.2017.05.029.

Colina, M., Ayala,A., Rincón, D., Molina, J., Medina, J., Yncarte, R., Vargas, J. \& Montilla, B. (2014). Evaluación de los procesos para la obtención química de quitina y quitosano a partir de desechos de cangrejos. Escala piloto e industrial. Revista Iberoamericana de Polímeros, 15(1), 21-43. http:// www.ehu.eus/reviberpol/pdf/Ene14/colina.pdf.

Collins, F., Rozhkovskaya, A., Outram, J. G. \& Millar, G. J. (2020). A critical review of waste resources, synthesis, and applications for Zeolite LTA. Microporous and Mesoporous Materials, 291,109667. https://doi.org/10.1016/j. micromeso.2019.109667.

Corvo, Y. H., López, E. B., León, V. \& Corrales, Y. A. (2014). Obtención de una matriz polimérica a base de celulosa para la adsorción de metales pesados. Revista Iberoamericana de Polimeros, 15(2), 75-84. http://www.ehu.eus/reviberpol/ pdf/MAR14/hernandez.pdf.

Crisóstomo, V. M. B., Ngala, J. K., Alia, S., Dobley, A., Morein, C., Chen, C.-H., Shen, X. \& Suib, S.(2007). New Synthetic Route, Characterization, and Electrocatalytic Activity of Nanosized Manganite. Chemistry of Materials, 19(7), 1832-1839. https://doi.org/10.1021/cm062871z.

Dialynas E., Mantzavinos, D. \& Diamadopoulos, E. (2008). Advanced treatment of the reverse osmosis concentrate produced during reclamation of municipal wastewater, Water Research, 42, 4603-4608. https://doi.org/10.1016/j. watres.2008.08.008.

Edelstein, M. \& Ben-Hur, M. (2018). Heavy metals and metalloids: Sources, risks and strategies to reduce their accumulation in horticultural crops. Scientia Horticulturae, 234, 431-444. https://doi.org/10.1016/j. scienta.2017.12.039.

Escoda, A., Euvrard, M., Lakard, S., Husson, J., Mohamed, A. S. \& Knorr, M. (2013). Ultrafiltration-assisted retention of $\mathrm{Cu}$ (II) ions by adsorption on chitosan-functionalized colloidal silica particles. Separation and Purification Technology, 118, 25-32. https://doi.org/10.1016/j.seppur.2013.06.017.

Fernández-Constantino, O. (1998). La contaminación y las pequeñas industrias en México. Comercio Exterior, 12, 960-965. http://revistas.bancomext.gob.mx/rce/ magazines/353/3/RCE3.pdf.

González-García, P. (2018). Activated carbon from lignocellulosics precursors: A review of the synthesis methods, characterization techniques and applications. Renewable and Sustainable Energy Reviews, 82, 1393-1414. https://doi.org/10.1016/j.rser.2017.04.117.

Hong, M., Yu, L., Wang, Y., Zhang, J., Chen, Z., Dong, L., Zan, Q. \& Li, R. (2019). Heavy metal adsorption with zeolites: The role of hierarchical pore architecture. Chemical Engineering Journal A, 359, 363-372. https:// doi.org/10.1016/j.cej.2018.11.087.

Hong, T. T., Okabe, H., Hidaka, Y. \& Hara, K. (2018). Radiation synthesis and characterization of super-absorbing hydrogel from natural polymers and vinyl monomer. Environmental Pollution B, 242, 1458-1466. https://doi.org/10.1016/j. envpol.2018.07.129.

Ihsanullah Abbas, A.,Al-Amer, A. M., Laoui, T.,Al-Marri, M. J., Nasser, M. S., Kraisheh, M. \& Atieh, M. A. (2016) . Heavy 
metal removal from aqueous solution by advanced carbon nanotubes: Critical review of adsorption applications. Separation and Purification Technology, 157, 141-161. https://doi.org/10.1016/j.seppur.2015.11.039.

Lee, X. J., Hiew, B. Y. Z., Lai, K. C., Lee, L. Y., Gan, S., Thangalazhy-Gopakumar, S. \& Rigby, S. (2019). Review on graphene and its derivatives: Synthesis methods and potential industrial implementation. Journal of the Taiwan Institute of Chemical Engineers, 98, 163-180. https://doi. org/10.1016/j.jtice.2018.10.028.

Liu, P., Jiang, L., Zhu, L., Guo, J. \& Wang, A. (2015). Synthesis of covalently crosslinked attapulgite/poly (acrylic acid-coacrylamide) nanocomposite hydrogels and their evaluation as adsorbent for heavy metal ions. Journal of Industrial and Engineering Chemistry, 23, 188-193. https://doi. org/10.1016/j.jiec.2014.08.014.

Nierboer, E. \& Richardson, D. M. S. (1980). The replace of the nondescript term $<<$ heavy metals $>>$ by a biologically and chemically significant classification of metal ions. Environmental Pollution Series B, 1,3-26. https://doi. org/10.1016/0143-148X(80)90017-8.

Mitra, M., Mahapatra, M., Dutta, A., Roy, J. S. D., Karmakar, M., Deb, M., Mondal, H., Chattopadhyay, P. K., Bandyopadhyay, A. \& Singha, N. R. (2019). Carbohydrate and collagen-based doubly-grafted interpenetrating terpolymer hydrogel via $\mathrm{N}-\mathrm{H}$ activated in situ allocation of monomer for superadsorption of $\mathrm{Pb}$ (II), $\mathrm{Hg}$ (II), dyes, vitamin-C, and p-nitrophenol. Journal of Hazardous Materials, 369, 746-762. https://doi.org/10.1016/j. jhazmat.2018.12.019.

Noor, N. M., Othman, R., Mubarak, N. M. \& Abdullah, E. C. (2017). Agricultural biomass-derived magnetic adsorbents: Preparation and application for heavy metals removal. Journal of the Taiwan Institute of Chemical Engineers, 78, 168-177. https://doi.org/10.1016/j. jtice.2017.05.023.

Roy, C., Dutta, A., Mahapatra, M., Karmakar, M., Roy, J. S. D., Mitra, M., Chattopadhyay, P. K. \& Singha, N. R. (2019). Collagenic waste and rubber based resin-cured biocomposite adsorbent for high-performance removal(s) of $\mathrm{Hg}$ (II), safranine, and brilliant cresyl blue: A costfriendly waste management approach. Journal of Hazardous Materials, 369, 199-213. https://doi.org/10.1016/j. jhazmat.2019.02.004.

Shams, M., Dehghani, M. H., Nabizadeh, R., Mesdaghinia, A., Alimohammadi, M. \& Najafpoor, A.A. (2016). Adsorption of phosphorus from aqueous solution by cubic zeolitic imidazolate framework-8: Modeling, mechanical agitation versus sonication. Journal of Molecular Liquids, 224, 151157. https://doi.org/10.1016/j.molliq.2016.09.059.

Shannon, M. A., Bohn, P. W., Elimelech, M., Georgiadis, J. G., Mariñas, B. J. \& Mayes, A. M. (2008). Science and technology for water purification in the coming decades. Nature, 452,301-310. https://doi.org/10.1038/nature06599.
Siddiqui, S. I., Naushad, Mu \& Chaudhry, S. A. (2019). Promising prospects of nanomaterials for arsenic water remediation: A comprehensive review. Process Safety and Environmental Protection, 126, 60-97. https://doi. org/10.1016/j.psep.2019.03.037.

Singh, N. B., Nagpal, G., Agrawal, S. \& Rachna. (2018). Water purification by using Adsorbents: AReview. Environmental Technology \& Innovation, 11, 187-240. https://doi. org/10.1016/j.eti.2018.05.006.

Sönmezay, A., Öncel, M. S. \& Bektaş, N. (2012). Adsorption of lead and cadmium ions from aqueous solutions using manganoxide minerals. Transactions of Nonferrous Metals Society of China, 22(12), 3131-3139. https://doi. org/10.1016/S1003-6326(12)61765-8.

Vera, C. C., Pérez, N. \& Sabino, M. (2016). Efecto de la cantidad de fase interpenetrada lignocelulósica y la composición sobre el proceso de hinchamiento y síntesis de hidrogeles interpenetrados en base a acrilamida. Revista Iberoamericana de Polímeros, 17(4), 170-182. http:/www. ehu.eus/reviberpol/pdf/JUL16/chacon.pdf.

Vieira, R. M., Vilela, P. B., Becegato, V. A. \& Paulino, A. T. (2018). Chitosan-based hydrogel and chitosan/acidactivated montmorillonite composite hydrogel for the adsorption and removal of $\mathrm{Pb}^{+2}$ and $\mathrm{Ni}^{+2}$ ions accommodated in aqueous solutions. Journal of Environmental Chemical Engineering, 6(2), 2713-2723. https://doi.org/10.1016/j. jece.2018.04.018.

Waheed, A., Mansha, M. \& Ullah, N. (2018). Nanomaterialsbased electrochemical detection of heavy metals in water: Current status, challenges and future direction. $\operatorname{Tr} A C$ Trends in Analytical Chemistry, 105, 37-51. https://doi. org/10.1016/j.trac.2018.04.012.

Wang, J., Wei, L., Ma, Y., Li, K., Li, M., Yu, Y., Wang, L. \& Qiu, H. (2013a). Collagen/cellulose hydrogel beads reconstituted from ionic liquid solution for $\mathrm{Cu}$ (II) adsorption. Carbohydrate Polymers, 98(1), 736-743.https:// doi.org/10.1016/j.carbpol.2013.06.001.

Wang, W., Zong, L. \& Wang, A. (2013b). A nanoporous hydrogel based on vinyl-functionalized alginate for efficient absorption and removal of $\mathrm{Pb}^{+2}$ ions. International Journal of Biological Macromolecules, 62, 225-231. https://doi. org/10.1016/j.ijbiomac.2013.08.038.

Wang, J., Yue, X., Yang, Y., Sirisomboonchai, S., Wang, P., Ma, X., Abudula, A. \& Guan, G. (2020). Earth-abundant transition-metal-based bifunctional catalysts for overall electrochemical water splitting: A review. Journal of Alloys and Compounds, 819, 153346. https://doi.org/10.1016/j. jallcom.2019.153346.

Wanna, Y., Chindaduang, A., Tumcharern, G., Phromyothin, D., Porntheerapat, S., Nukeaw, J., Hofmann, H. \& Pratontep, S. (2016). Efficiency of SPIONs functionalized with polyethylene glycol bis(amine) for heavy metal removal. Journal of Magnetism and Magnetic Materials, 414, 32-37. https://doi.org/10.1016/j.jmmm.2016.04.064. 
Wen, J., Fang, Y. \& Zeng, G. (2018). Progress and prospect of adsorptive removal of heavy metal ions from aqueous solution using metal-organic frameworks: A review of studies from the last decade. Chemosphere, 201, 627-643. https://doi.org/10.1016/j.chemosphere.2018.03.047.

Xu, R., Zhou, G., Tang, Y., Chu, L., Liu, C., Zeng, Z. \& Luo, S. (2015). New double network hydrogel adsorbent: Highly efficient removal of Cd (II) and Mn (II) ions in aqueous solution. Chemical Engineering Journal, 275, 179-188. https://doi.org/10.1016/j.cej.2015.04.040.
Xue, X., Cheng, R., Shi, L., Ma, Z. \& Zheng, X. (2017). Nanomaterials for water pollution monitoring and remediation. Environmental Chemistry Letters, 15(1), 2327. https://doi.org/10.1007/s10311-016-0595-X.

Yu, J. W., Jung, J., Choi, Y.-M., Choi, J. H., Yu, J., Lee, J. K., You, N. H. \& Goh, M. (2016). Enhancement of the crosslink density, glass transition temperature, and strength of epoxy resin by using functionalized graphene oxide cocuring agents. Polymer Chemistry, 7(1), 36-43. https://doi. org/10.1039/C5PY01483B. 